\title{
Prostatic artery embolization performed in anteroposterior projections versus steep oblique projections: single centre retrospective comparative analysis
}

Hippocrates Moschouris ${ }^{1 *}$ (D), Andreas Dimakis ${ }^{1}$, Marina G. Papadaki ${ }^{1}$, Athanasios Liarakos ${ }^{2}$, Konstantinos Stamatiou $^{3}$, Ioulita Isaakidou', Ilianna Tsetsou', Vasiliki Mylonakou' and Katerina Malagari ${ }^{4}$

\begin{abstract}
Background: To present and evaluate an approach for reduction of utilization of steep oblique angiographic projections during prostatic artery embolization (PAE).

Methods: Single-center, retrospective study of patients who underwent bilateral PAE (from October 2018 to November 2019) and in whom it was possible to embolize PA of at least one pelvic side utilizing anteroposterior projections only (AP-PAE group), with the following techniques: Identification of the origin of PA on anteroposterior angiographic views. Utilization of anatomic landmarks from the planning computed tomographic angiography. Distal advancement of the angiographic catheter or microcatheter in the anterior division of internal iliac artery. Gentle probing with microguidewire at the expected site of origin of the PA. The AP-PAE approach was initially applied to all PAE patients during the study period and when this approach failed, additional steep oblique projections were acquired; patients who underwent bilateral PAE, with both anteroposterior and oblique projections for both pelvic sides, formed the standard PAE (S-PAE) group. The AP-PAE group was compared with S-PAE group in terms of baseline clinical and anatomic features, technical/procedural aspects and outcomes.

Results: Forty-six patients (92 pelvic sides) were studied. AP-PAE was feasible in 12/46 patients (26.0\%): unilateral APPAE in 9/46 patients (19.5\%); bilateral AP-PAE in $3 / 46$ patients (6.5\%). AP-PAE group had larger prostates $(p=0.047)$ and larger PAs $(p<0.001)$. Body mass index (BMI) and other baseline features were comparable between the two groups (mean BMI, AP-PAE group: $27.9 \pm 3.6$, S-PAE group: $27.0 \pm 3.5, p=0.451)$. Mean fluoroscopy time and dose area product were lower in AP-PAE group (46.3 vs $57.9 \mathrm{~min}, p=0.084$ and 22,924.9 vs $35,800.4 \mu \mathrm{Gy}^{2} \mathrm{~m}^{2}, p=0.018$, respectively). Three months post PAE, comparable clinical success rates $(11 / 12$ vs $31 / 34, p=0.959)$ and mean International Prostate Symptom Score reduction $(60.2 \%$ vs $58.1 \%, p=0.740)$ were observed for AP-PAE and for S-PAE group, respectively. No major complications were encountered.
\end{abstract}

Conclusion: AP-PAE is associated with significant reduction in radiation exposure and appears to be feasible, safe and effective, but it can be applied in a relatively small percentage of patients.

Keywords: Prostatic artery embolization, Anteroposterior projections, Oblique projections, Pelvic angiography, Dose area product

\footnotetext{
* Correspondence: hipmosch@gmail.com

'Radiology Department, General Hospital "Tzanio", Zanni \& Afentouli 1 Str., 18536 Piraeus, Greece

Full list of author information is available at the end of the article
}

\section{Springer Open}

(c) The Author(s). 2021 Open Access This article is licensed under a Creative Commons Attribution 4.0 International License, which permits use, sharing, adaptation, distribution and reproduction in any medium or format, as long as you give appropriate credit to the original author(s) and the source, provide a link to the Creative Commons licence, and indicate if changes were made. The images or other third party material in this article are included in the article's Creative Commons licence, unless indicated otherwise in a credit line to the material. If material is not included in the article's Creative Commons licence and your intended use is not permitted by statutory regulation or exceeds the permitted use, you will need to obtain permission directly from the copyright holder. To view a copy of this licence, visit http://creativecommons.org/licenses/by/4.0/. 


\section{Introduction}

Prostatic artery embolization (PAE) is a technically challenging endovascular procedure due to the variant pelvic arterial anatomy, frequent tortuosity and atheromatosis of these arteries, small size of prostatic arteries (PAs) and frequent anastomoses and overlap with arteries of neighbouring organs (Bilhim et al. 2012a, 2012b; Carnevale et al. 2017). To facilitate identification and catheterization of PAs, steep oblique fluoroscopic and angiographic views (ipsilateral oblique at $35^{\circ}-45^{\circ}$ with additional caudalcranial angulation of approximately $10^{\circ}$ ) are routinely utilized as a standard step of the PAE procedure (Bilhim et al. 2012a, 2012b, Carnevale et al. 2017). Thanks to these projections, most of the pelvic arterial branches relevant to PAE (Pudendal, Rectal, Obturator, Vesical Inferior and Superior, under the ipsilateral Oblique view-"PROVISO" acronym, Carnevale et al. 2017) can be identified with relative ease and with limited superposition of other pelvic arteries.

However, compared to anteroposterior, steep oblique views are associated with a significantly increased radiation dose to patient and staff. Of note, pelvic endovascular procedures are accompanied by significantly higher effective doses for the operator than peripheral vascular interventions and this difference is largely attributable to the frequency of oblique projections during the former (Ingwersen et al. 2013). In a recent systematic review focusing on radiation exposure during PAE (Zumstein et al. 2020), wide variations in dose-area product (DAP) between different PAE studies were found (mean DAP: 3316-86,340 $\mu \mathrm{Gy}^{\prime}{ }^{2}$ ) and the large potential to reduce this exposure was emphasized. Optimization (meaning that imaging should be performed using doses that are as low as reasonably achievable - "ALARA") is one of the three main principles of radiation protection (Hertault et al. 2015; Do 2016); it would probably be worth investigating how and if reduction of oblique views could facilitate the implementation of the ALARA principle in the context of PAE.

In practice, in some cases of PAE, PA of at least one pelvic side can be selected and embolized using exclusively anteroposterior fluoroscopic and angiographic imaging. In this report, a series of such cases is reviewed and the relevant technical steps are described. The feasibility, efficacy, safety and potential benefits of this approach (PAE with anteroposterior angiographic imaging only) are evaluated and compared with standard technique of PAE.

\section{Materials and methods}

Institutional review board approval was obtained for this study.

\section{Patients, equipment and consent}

Patients who were treated with bilateral PAE for symptomatic benign prostatic hyperplasia at a single institution during (approximately) a 1-year period, from October 2018 to November 2019, were retrospectively reviewed. Pre-procedural evaluation, inclusion and exclusion criteria were the same with previous work (Moschouris et al. 2019) and with other PAE studies (Pisco et al. 2016). To eliminate the effects of the learning curve, patients treated during the first 2 years of the local PAE practice (July 2016-September 2018) were excluded. Also excluded were cases of unilateral or failed $\operatorname{PAE}$ ( $n=6$ and $n=2$, respectively) during the study period and procedures which were performed after November 2019, with newer equipment (Axiom Artis Zee, Siemens Healthineers, Erlangen, Germany, instead of Axiom Artis MP, Siemens, which was utilized for all the patients of this study).

Written informed consent was obtained from all patients prior to treatment.

\section{Technique}

Pre-procedural clinical and imaging evaluation was performed according to the institute's standard protocol (Moschouris et al. 2019). Computed-Tomographic Angiography (CTA) was performed for treatment planning to all patients prior to PAE (Table 1 of supplementary material). The origin and course of the PAs was studied on axial CT slices and on Maximum Intensity Projections (MIPs) on coronal, sagittal and oblique sagittal planes. Anatomic landmarks that facilitated localization of the origin of PAs on anteroposterior MIP projections were noted, for subsequent correlation with anteroposterior pelvic digital subtraction angiography (DSA). For each pelvic side, the type of origin of PA was defined according to the classification system (Fig.1), proposed by de Assis et al. (2015). The diameter of the PA was measured at its origin. The tortuosity of the pelvic arteries was assessed and graded, using a practical categorization of a previous PAE study (Enderlein et al. 2020): Grade 1 (mild: kinking $<30^{\circ}$ in both pelvic sides), Grade 2 (moderate: maximum kinking $30^{\circ}-60^{\circ}$ in at least 1 pelvic side), and Grade 3 (severe: multiple kinking $30^{\circ}{ }^{\circ}-60$ ${ }^{\circ}$ in both sides and kinking of $>60^{\circ}$ in at least 1 pelvic side). Angles were measured in coronal MIP images. Finally, in line with a previous report (Hacking et al. 2019), the diameter of the internal iliac arteries was visually assessed and atheromatous stenoses were classified as: Grade 0, 1, 2 and 3 (for no, mild, moderate and severe stenoses, respectively).

With the exception of patients with indwelling bladder catheter (IBC), PAE was performed without previous bladder catheterization. Vascular access was obtained via the right common femoral artery or left radial artery. 
The latter access (preceded by the Barbeau test and preferred when preprocedural CTA indicated severe tortuosity and/or significant stenoses of the external iliac and femoral arteries) entailed ultrasonographically guided cannulation of the radial artery $1-2 \mathrm{~cm}$ proximal to the radial styloid process and utilization of an appropriate introducer (Prelude radial sheath introducer, Merit Medical, South Jordan, UT, USA). The internal iliac arteries (IIAs) were catheterized with a 5 French (Fr.) angiographic catheter. In case of femoral access, $65-80 \mathrm{~cm}$ double angle or reverse curve catheters (Cobra1, Simmons 1, Contra 2 or Uterine Artery catheter, Merit Medical, or Boston Scientific, MA, USA,) were utilized for the ipsilateral IIA. If catheterization of the contralateral IIA could not be achieved with these catheters, they were exchanged for a hydrophilic one (Vertebral Glidecath, Terumo Corporation, Japan). In case of left radial access, an $125 \mathrm{~cm}$ Multipurpose or Vertebral catheter (Merit Medical) was utilized.

Anteroposterior DSA of the IIAs was performed with manual injection of $10-20 \mathrm{ml}$ of contrast through the angiographic catheter. One or more of the following techniques were applied, in order to perform PAE with anteroposterior imaging only (AP-PAE): 1) Identification of the origin of PA on DSA and subsequent attempt for catheterization, usually with the aid of a roadmap. 2) Utilization of anatomic landmarks from the planning CTA, in order to approach the origin of the PA. 3) Distal advancement of the microcatheter/microguidewire or (preferably) advancement of the 5Fr. angiographic catheter over the hydrophilic guidewire in the anterior division of IIA, far from the origins of superior and inferior gluteals and closer to the expected PA origin. Anteroposterior angiograms performed from this distal position depicted the target vessels without superposition of the muscular (superior/inferior gluteal) branches and facilitated identification of the PA. 4) Gentle probing with microcatheter and microguidewire at the expected site of origin of the PA. A common combination was a microcatheter with angled tip (Direxion 2.4Fr, Bern shape, Boston Scientific) and a double-angled 0.016"microguidewire (Meister- Asahi Intecc co, Japan).

No more than 3-5 min of operation time and approximately $1 \mathrm{~min}$ of fluoroscopy per pelvic side were devoted to these attempts. If they proved fruitless, steep oblique DSAs were acquired (25- $45^{\circ}$ with additional caudalcranial angulation $8-12^{\circ}$,taking into account the angle of oblique MIPs that optimized detection of PA origin), as per standard PAE technique (S-PAE).

Prostatic arteries were subsequently catheterized with the microcatheter and microguidewire; nitroglycerin $(250 \mu \mathrm{g})$ was administered through the microcatheter for vasodilation and additional angiograms (only anteroposterior, in case of AP-PAE) were performed with manual injection of 2-4 $\mathrm{ml}$ of contrast through the microcatheter. Embolization was started at the (preferably distal) extraprostatic part of the PA, after advancement of the microcatheter beyond the potential origin of collateral branches to the bladder, rectum or penis. When complete flow stasis was observed, advancement of the microguidewire (followed by the microcatheter) into the intraprostatic branches was attempted ("PErFecTED" technique: "Proximal Embolization First, Then Embolize Distal", Carnevale et al. 2014).PAE was performed with microspheres (Embozene- Boston Scientific, or Embosphere-Merit Medical) with diameters of $100-500 \mu \mathrm{m}$. Prerequisites for selection of smaller microspheres (Embozene 250, or Embosphere 100-300) were: i) Advancement of the microcatheter at the distal extraprostatic part of the PA, or into the prostate. ii) Absence of large intraprostatic anastomoses with penile or rectal branches. iii) Safe distance of the

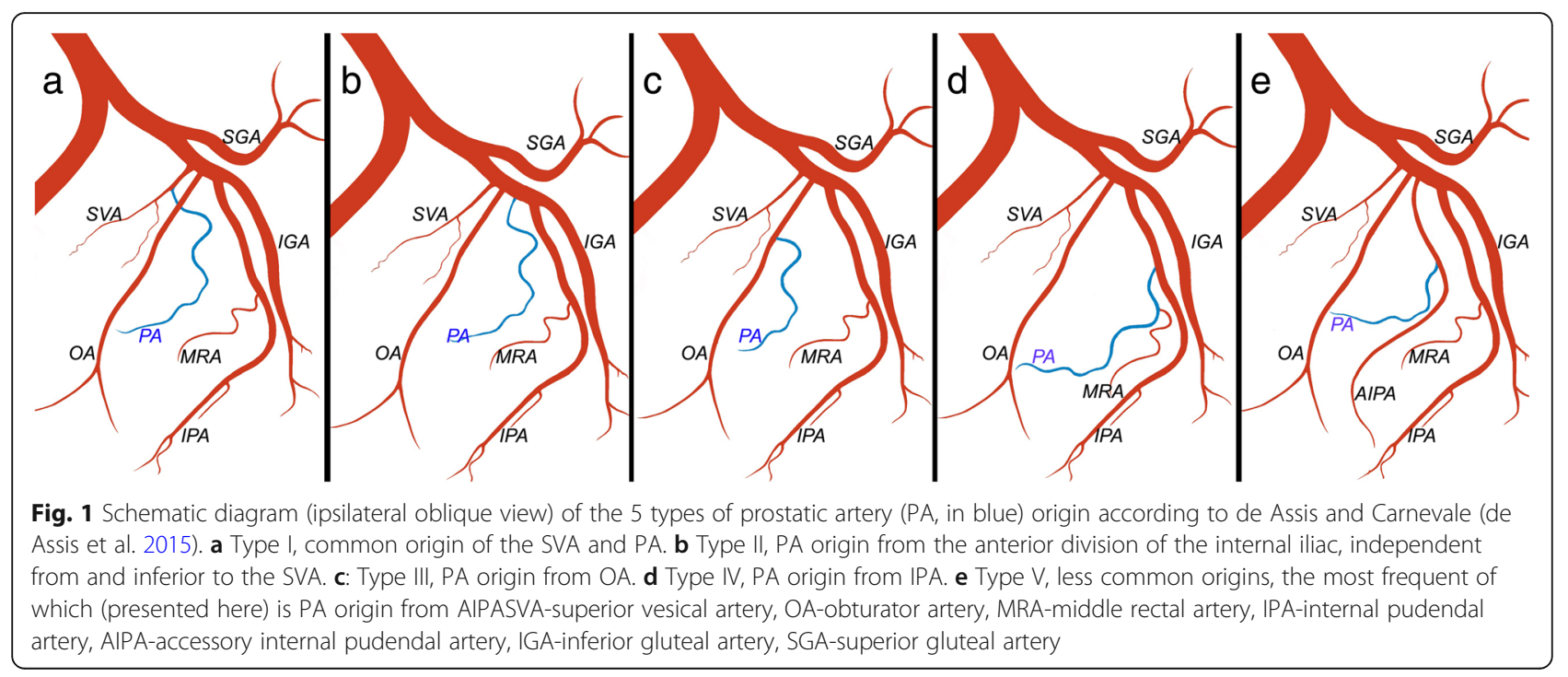


microcatheter tip from the origins of vesical, penile or rectal arteries. All other cases were treated with Embozene 400, or Embosphere 300-500 microspheres. Selection of the one manufacturer over the other depended on the material availability at the time of intervention.

For all PAE procedures the same angiographic protocol of the equipment ('General DSA') was selected. All DSA acquisitions were performed at 1 frame/second ( $\mathrm{fr} /$ s) and fluoroscopy at $7.5 \mathrm{fr} / \mathrm{s}$. Additional exposure parameters are provided in Table 2 of supplementary material. A Cone-Beam Computed Tomography (CBCT) facility was not available during the study period. Intraprocedural contrast-enhanced ultrasonography (CEUS), with a second-generation echo enhancer (SonoVue, Bracco, Milan, Italy), with a portable unit (M8 Mindray, Nanshan, Shenzhen, China) was utilized to depict the area fed by the catheterized vessel and to evaluate the ischemic effect immediately post PAE (Moschouris et al. 2020). All procedures were performed by two interventional radiologists, each with 2 years of previous experience in PAE and more than 12 years in other pelvic embolization procedures. Postoperative care was similar to previous work (Moschouris et al. 2019). In case of transradial access, patients were discharged 5-6h post PAE.

\section{Evaluation of procedural parameters, efficacy and safety}

After each PAE procedure, several parameters (fluoroscopy time, dose-area product-DAP, number of acquisitions with the respective $\mathrm{C}$-arm projections, field size, irradiation parameters) were recorded by the angiographic unit and were extracted for analysis.

For the purposes of this study, a limited, short-term evaluation of imaging and clinical outcomes was performed. Transabdominal CEUS was performed in all patients $18-24 \mathrm{~h}$ post PAE to measure the extent of prostatic infarction. Transabdominal US was performed 3 months post PAE and changes (compared to baseline measurements) in prostate volume and post-void residual were recorded Further details on sonographic intra- and postprocedural monitoring of embolization are provided in Table 3 of supplementary material. The International Prostate Symptom Score (IPSS) was also calculated 3 months post PAE and compared with baseline. Clinical success was defined as an IPSS $\leq 15$ points with a decrease of at least $25 \%$ from the baseline and with no need for additional medical or surgical treatment post PAE. For patients with IBC, clinical success was defined as permanent catheter removal with spontaneous micturition and post void residual (PVR) $<100$ $\mathrm{ml}$. In these patients, trials of catheter removal were performed every week for the first month post PAE and every 2 weeks for the second month.

\section{Comparisons and statistical analysis}

Patients who underwent bilateral embolization, with APPAE of at least one pelvic side formed the AP-PAE group; patients who underwent bilateral embolization with standard technique (first with anteroposterior and then with oblique imaging of both pelvic sides) formed the S-PAE group. Descriptive statistics were calculated for quantitative and qualitative data for both groups. Differences in baseline characteristics, procedural, technical parameters and outcomes between the two groups were evaluated. Particularly for the S-PAE group, comparable anteroposterior and oblique angiographic runs (of the same anatomic area and with the same frame number and collimation) were identified and the respective DAPs were recorded. Several tests were used for comparisons, depending on the kind, sample size and distribution of each variable (t-test, Welch test, Mann-Whitney U test, Chi-Square test). Statistical significance was defined as a $p$ value of $<0.05$.

\section{Results}

Forty-six patients (92 pelvic sides) were studied. AP-PAE group included $12 / 46$ patients (26\%). Unilateral AP-PAE could be achieved in 9/12 patients (19.5\% of all patients of the study). In unilateral AP-PAE, the contralateral pelvic side was embolized after additional oblique imaging, as per standard technique. Bilateral AP-PAE was performed in $3 / 12$ patients $(6.5 \%$ of all patients of the study), resulting in a total of $15 / 92(16.3 \%)$ pelvic sides which were embolized with utilization of anteroposterior imaging only (Figs 1, 2 of supplementary material). No cases of dissection or other iatrogenic vascular injury were observed during the manoeuvres of AP-PAE.

\section{Anatomic features of the two groups}

Compared to the S-PAE group (34/46 patients), AP-PAE group had significantly larger prostates $(p=0.047)$ and larger prostatic arteries $(p<0.001)$. Other baseline demographic, clinical and most of the anatomic features were comparable between the two groups (Table 1).

Regarding the origin of PA, type IV (prostatic artery origin from the internal pudendal) was far commoner among the pelvic sides treated with AP-PAE (6/15 pelvic sides or $40 \%$, versus $15 / 77$ or $19.5 \%$ of the pelvic sides treated with S-PAE, $p=0.085)$. On the contrary, type I (common origin of the superior vesical and inferior vesical-prostatic artery) was far commoner among the pelvic sides treated with S-PAE (34/77 pelvic sides or $44.1 \%$, versus $3 / 15$ or $20 \%$ of the pelvic sides treated with AP-PAE, $p=0.082$ ). The prevalence of type III (prostatic artery origin from the obturator artery) was slightly higher among pelvic sides of AP-PAE and type II (prostatic artery origin from the anterior division of the internal iliac independent from and inferior to the 
superior vesical) was slightly more common among pelvic sides of S-PAE (Table 4 of supplementary material). In one of the 15 pelvic sides treated with AP-PAE, PA originated from accessory pudendal artery (type V). We also observed 2 type $\mathrm{V}$ origins in the 77 pelvic sides treated with standard technique (from accessory pudendal artery, $n=1$, and from trifurcation of anterior division, $n=1$ ). There was one case of AP-PAE, in which planning CTA indicated PAE duplication, but only one of the 2 PAs could be identified and catheterized during the procedure. On the other hand, during S-PAE, 2 cases of PA duplication were correctly recognized and appropriately managed. Two cases of anastomoses of PA with middle rectal artery (MRA) could be identified during AP-PAE; in both, it was possible to advance the microcatheter distal to the origin of the MRA.

\section{Radiation and other procedural data}

Mean fluoroscopy time was $20 \%$ shorter in AP-PAE group, although the difference did not reach statistical significance $(p=0.084)$. However, the mean DAP of AP-PAE was $35.9 \%$ lower compared to S-PAE (22, 924.9 vs $35,800.4 \mu \mathrm{Gy}^{2} \mathrm{~m}^{2}$ and this difference was statistically significant $(p=0.018$, Table 2$)$. In the S-PAE group, 52 pairs of comparable anteroposterior and steep oblique angiographic runs were identified. The latter were associated with a significantly higher mean DAP $\left(1255.7 \pm 822.7 \mu \mathrm{Gy}^{\prime} \mathrm{m}^{2}\right.$ vs $683.7 \pm 479.4 \mu \mathrm{Gy}^{\prime} \mathrm{m}^{2}$, mean difference: $94.2+/-76.4 \%, p=0.001)$. Additional technical procedural details are provided in Table 5 of supplementary material.

\section{Outcomes and follow up}

On early ( 1 day post PAE) CEUS evaluation, the two approaches resulted in comparable extent of prostatic infarction. Three months post PAE, clinical success rates were 91.6\% (11/12 patients) versus 91.1\% (31/34 patients) for AP$\mathrm{PAE}$ and for S-PAE group, respectively $(p=0.959)$. The rates of international prostate symptom score (IPSS) reduction and of PV and PVR reduction were also similar for the two groups (Table 3). Only minor complications were observed in 5/34 patients of the S-PAE group (acute urinary retention: $n=3$, small groin hematoma: $n=2$ ) and in 3/12 patients of the AP-PAE group (acute urinary retention: $n=1$, small forearm hematoma post transradial access: $n=1$, transient, self-limiting hemospermia: $n=1$ ). Differences in prevalence of complications between the 2 groups were not significant $(p=0.440)$.

\section{Discussion}

This study showed that the AP-PAE approach was associated with a reduction of oblique fluoroscopic and angiographic projections in a relatively small but not negligible percentage (26\%) of patients. AP-PAE required only moderate experience, no novel techniques and no specialized equipment. In the short-term, imaging and clinical outcomes of this approach were satisfactory and comparable to those of S-PAE.

Several factors may have contributed to technical success of AP-PAE:

Pelvic CTA has an established role in facilitating planning of original PAE (Bilhim et al. 2012a, 2012b) and it also proved indispensable in the context of AP-PAE. Coronal MIP reconstructions were particularly useful to identify the origin of PA preoperatively and to guide the attempts for catheterization of the PA (or, at least, for catheterization of its parent vessel).

Additionally, the herein reported results are largely attributed to some favorable anatomic aspects of the APPAE group. These patients had significantly larger prostates and larger diameters of the PAs and these two features are considered as predictors of technical success of PAE (Enderlein et al. 2020; Hacking et al. 2019; Lintin et al. 2020). Large prostates are fed by large prostatic arteries, which are easier to identify (even when imaging is

Table 1 Baseline demographic, anatomic and clinical data for the two patient groups of this study

\begin{tabular}{llll}
\hline & S-PAE $(\boldsymbol{n = 3 4 )}$ & AP-PAE $(\boldsymbol{n}=\mathbf{1 2})$ & $\boldsymbol{P}$-value \\
\hline Age (yrs), (mean \pm SD, years) & $73.3 \pm 7.9$ & $73.9 \pm 9.7$ & 0.832 \\
BMI, (mean \pm SD) & $27.0 \pm 3.5$ & $27.9 \pm 3.6$ & 0.451 \\
PV (mean \pm SD, ml) & $89.5 \pm 27.4$ & $111.4 \pm 42.8$ & $0.047^{*}$ \\
PA diameter (mean \pm SD, mm) & $1.4 \pm 0.2$ & $1.7 \pm 0.2$ & $<0.001^{*}$ \\
Tortuosity Index (mean \pm SD) & $2.3 \pm 0.8$ & $1.9 \pm 0.8$ & 0.152 \\
Stenosis index (mean \pm SD) & $1 \pm 0.42$ & $1 \pm 0.94$ & 1.000 \\
LUTS, proportion of pts & $25 / 34$ & $9 / 12$ & 0.921 \\
IPSS (mean $\pm S D)$ & $25.5 \pm 5.6$ & $28.8 \pm 3.3$ & 0.107 \\
PVR (mean $\pm S D, m l)$ & $158 \pm 62$ & $143 \pm 87$ & 0.580 \\
IBC (\% of pts) & $26.5(n=9)$ & $25(n=3)$ & 0.921 \\
\hline
\end{tabular}

SD standard deviation, $B M I$ body mass index, $P V$ prostate volume, PA prostate artery, LUTS lower urinary tract symptoms, IPSS international prostate symptom score, $P V R$ post void residual, $I B C$ indwelling bladder catheter

*statistically significant 
Table 2 Comparison of radiation dose - related features of PAE for the two groups

\begin{tabular}{llll}
\hline & S-PAE $(\boldsymbol{n}=\mathbf{3 4})$ & AP-PAE $(\boldsymbol{n}=\mathbf{1 2})$ & \multicolumn{1}{c}{$\boldsymbol{P}$-value } \\
\hline Fluoroscopy time $($ mean $\pm \mathrm{SD}, \mathrm{min})$ & $57.9 \pm 21.7$ & $46.3 \pm 11.1$ & 0.084 \\
DAP $\left(\right.$ mean $\left.\pm \mathrm{SD}, \mu \mathrm{Gy}^{2}{ }^{2}\right)$ & $35,800.4 \pm 16,819.9$ & $22,924.9 \pm 11,305.9$ & $0.018^{*}$ \\
Number of runs per session & $23.6 \pm 6.5$ & $20.3 \pm 7.4$ & 0.188 \\
Number of images per session & $240.7 \pm 66,3$ & $217.2 \pm 79.1$ & 0.370 \\
Contribution of acquisitions vs fluoroscopy in DAP $(\%)$ & $60.6 / 39.4$ & $52.1 / 47.9$ & 0.226 \\
\hline
\end{tabular}

SD standard deviation, DAP dose area product

* statistically significant

limited to anteroposterior views) and catheterize. This results in shorter fluoroscopy times, (perhaps more importantly) reduced need for oblique views and, eventually, reduced radiation dose to patient and operator. On the contrary, tortuosity of the pelvic arteries has negative effect on technical outcomes of PAE (Lintin et al. 2020), although for this predictor, the difference in favor of AP-PAE group was not statistically significant.

It is also likely, that technical success of AP-PAE (and the subsequent benefit in terms of reduced dose). depended on the type of origin of PA. Types III and (particularly) IV were more common in AP-PAE than in S-PAE. It was relatively easy to catheterize the obturator or internal pudendal without resorting to oblique views; on subsequent DSA, it was also relatively straightforward to identify the origin of PA from the respective arteries. On the contrary, greater difficulties were experienced in identifying and selecting type I and II origin of PA, and the prevalence of these types was comparatively lower in AP-PAE group. In the context of PAE with the original technique, other researchers (Enderlein et al. 2020; Eldem et al. 2020) have also found a positive effect of type III and IV origins on technical success and on shorter procedure time, whereas type I was a predictor of technical failure of bilateral PAE (Bilhim et al. 2013). Finally, one of the cases with rarer type of PA origin (type V) as well as duplicate PAs could be treated only with S-PAE. This probably indicates that complex and less frequent variants of PA anatomy may not be suitable for AP-PAE.

The present study showed that AP-PAE was associated with a significantly lower (35.9\%) mean DAP compared to S-PAE, and this is probably the most important advantage of the former approach. DAP is defined as the incident dose multiplied by the surface area irradiated; DAP represents the entire amount of energy delivered to the patient by the beam and is used as an indirect measure for assessing radiation risk, particularly for stochastic effects (Zumstein et al. 2020; Andrade et al. 2017; Hertault et al. 2015). Taking into account that other determinants of DAP (such as the BMI, operator, equipment and technical parameters) were the same (or not significantly different) between the two groups, the reduced radiation dose in AP-PAE should be attributed to the limited utilization of steep oblique fluoroscopic and angiographic projections.

Since PAE often requires long fluoroscopy times and numerous DSA runs at several projections, concerns have been raised about radiation safety of this procedure. In an elaborate study (Andrade et al. 2017) of several (direct and indirect) radiation parameters, PAE was associated with a greater peak skin dose to the patient (2420.3 mGy) than other complex endovascular procedures. The average effective dose per procedure for the interventional radiologist $(17 \mu \mathrm{Sv})$ was also comparable with other high-exposure interventions. Oblique projections were considered a major cause for the high dose

Table 3 Comparison of outcome parameters for the two groups

\begin{tabular}{|c|c|c|c|}
\hline & S-PAE $(n=34)$ & AP-PAE $(n=12)$ & $P$-value \\
\hline Percentage of prostatic infarction ${ }^{\mathrm{a}}$ (mean $\left.\pm \mathrm{SD}, \%\right)$ & $32.2 \pm 20.7$ & $28.0 \pm 11.7$ & 0.510 \\
\hline $\mathrm{PV}$ reduction ${ }^{\mathrm{b}}($ mean $\pm \mathrm{SD}, \%)$ & $26.4 \pm 15.4$ & $33.0 \pm 7.6$ & 0.062 \\
\hline IPSS reduction ${ }^{b}($ mean $\pm S D, \%)$ & $58.1 \pm 15.9$ & $60.2 \pm 16.0$ & 0.740 \\
\hline PVR reduction ${ }^{\mathrm{b}}($ mean $\pm \mathrm{SD}, \%)$ & $65 \pm 42$ & $56 \pm 50$ & 0.638 \\
\hline Clinical success ratio ${ }^{\mathrm{b}}(\%)$ & $91.1(n=3)$ & $91.6(n=1)$ & 0.959 \\
\hline Complications ${ }^{c}$-proportion of pts & $5 / 34$ & $3 / 12$ & 0.423 \\
\hline
\end{tabular}

$S D$ standard deviation, $P V$ prostate volume, IPSS international prostate symptom score, $P V R$ post void residual

${ }^{\text {a }}$ calculated 1 day post PAE

${ }^{b}$ calculated 3 months post PAE

'only minor complications were observed 
measurements; in line with these observations, the present study showed that in the S-PAE group, the mean DAP of steep oblique angiographic runs was almost twice as high as the mean DAP of comparable anteroposterior runs. Oblique projections are associated with increased scatter radiation, as a result of the increased amount of tissue that needs to be traversed by the $\mathrm{X}$ rays. Of note, an exponential increase of scattered radiation is observed when steep $\left(>30^{\circ}\right)$ right or left oblique views are applied (Hertault et al. 2015). Radiation exposure of the staff is further increased by the proximity of the X-ray tube to the operator, with left anterior obliquity resulting in higher exposure for an operator standing at the right side of the patient and vice-versa (Sukupova et al. 2018). Finally, steep oblique views may interfere with correct arrangement of the movable lead shields. Garzón et al. (2016) found that the combination of left anterior oblique projections and inappropriate positioning of ceiling suspended lead shield during PAE could result in high radiation doses to the eyes of the operator. If utilization of lead glasses had also been neglected, the annual equivalent dose limit for the eyes would have been reached with just one PAE procedure per week.

Taken together, all these data emphasize the need for careful and systematic application of the ALARA principle in the clinical context of PAE; strict adherence to the ALARA concept in other complex endovascular procedures has resulted in dramatic reduction of radiation exposure (Hertault et al. 2018). In selected patients, the herein proposed reduction of steep oblique views during PAE appears to be a feasible step towards the implementation of this principle.

A significant methodological limitation of this work should be acknowledged: Anteroposterior imaging was applied at the beginning of the procedure not only to the AP-PAE but also to the S-PAE group. Therefore, the increment in radiation exposure in the latter group could be partially attributed to the fact that S-PAE patients received both anteroposterior and oblique angiographic imaging. However, the initial anteroposterior imaging (applied to both groups) consisted only of a minute of fluoroscopy and one run per pelvic side, so its contribution to the overall radiation exposure of the SPAE group was small. Moreover, several operators utilize both anteroposterior and oblique projections in their standard PAE practice.

Additional limitations of the present study should also be mentioned: PAE was performed with older equipment, and the potential benefits of CBCT could not be evaluated, as this technique was not available. This was a retrospective study with small groups and short follow-up time. Only indirect measures of radiation dose were recorded, which are less accurate than direct ones (such as peak skin dose). Very few patients with significant pelvic arterial stenoses and no patients treated with unilateral PAE were studied, therefore AP-PAE was not tested in more technically challenging cases. For the same reason, the high clinical success rates reported in this study do not accurately reflect the clinical efficacy of AP-PAE under real-life conditions.

\section{Conclusions}

AP-PAE appears to be a feasible way to significantly reduce radiation exposure during PAE, with no compromise on safety and efficacy. However, the technique can be applied in a relatively small percentage of patients.

\section{Supplementary Information}

The online version contains supplementary material available at https://doi. org/10.1186/s42155-021-00209-7.

Additional file 1: Table 1. Basic parameters of pelvic CTA utilized for this study. Table 2. Exposure and other technical parameters of DSA.

Table 3. Basic features of the US modalities which were applied for evaluation of PAE. Table 4. Prevalence of different types of origin of PA for the two groups. Table 5. Comparison of technical features of PAE for the two groups. Figure 1. Application of AP-PAE in a case of type III origin of the right PA. Oblique sagittal (A), and anteroposterior (B), MIP reconstructions from the planning CTA show the obturator artery (open arrows) and the PA (arrows) originating from the proximal part of the obturator. Anteroposterior road-map image of the right IIA (C) and of its branches also shows the obturator artery (open arrow) and the PA (arrow), although the origin of the latter cannot be clearly identified. The obturator could be easily catheterized and selective angiography (D), with the microcatheter at its proximal part (open arrow), clearly showed the origin of the PA (arrow). Subsequent catheterization of the PA was also relatively easy (selective PA angiogram, E). Intraprocedural CEUS (F), after IV injection of SonoVue immediately post embolization of right PA, shows extensive devascularization of the right hemiprostate (asterisks). Figure 2. Application of AP-PAE in a case of bilateral type IV PA origin. Anteroposterior MIP reconstruction (A), from the planning CTA, shows PA of both sides (arrows) originating from the internal pudendals. Anteroposterior road-map image of the left IIA and of its branches (B), shows advancement of the microcatheter at the middle third of the internal pudendal (arrow). It was easy to proximally retract the microcatheter and then select the PA. Anteroposterior angiograms (C,D) show the tip of the microcatheter (arrows) in the extraprostatic part of the left PA. Anteroposterior angiogram of the right $\| \mathrm{A}$ and of its branches $(E)$, shows the origin of the right PA (open arrow) from the internal pudendal. Anteroposterior angiograms with the tip of the microcatheter at the origin and more distally in the right PA (arrows at F and G, respectively) show the typical appearance of the PA. Intraprocedural CEUS $(H)$, after IV injection of SonoVue at the end of the procedure, shows extensive devascularization of both prostatic lobes (asterisks)

\section{Abbreviations}

AP: Anteroposterior; BMI: Body mass index; CEUS: Contrast-enhanced ultrasonography; CTA: Computed tomographic angiography; DSA: Digital subtraction angiography; IBC: Indwelling bladder catheter; IIA: Internal iliac artery; IPSS: International prostate symptom score; IV: Intravenous; LUTS: Lower urinary tract symptoms; MIP: Maximum intensity projection; MRA: Middle rectal artery; PAE: Prostatic artery embolization; PA: Prostatic artery; PV: Prostate volume; PVR: Post void residual; S: Standard;

US: Ultrasonography

Acknowledgements

Not applicable. 


\section{Authors' contributions}

All authors read and approved the final manuscript.

\section{Funding}

There are no funding disclosures related to this work.

\section{Availability of data and materials}

The datasets used and/or analysed during the current study are available from the corresponding author on reasonable request.

\section{Ethics approval and consent to participate}

This retrospective study was approved by the ethics committee of our hospital. Written informed consent was obtained from patients to treat their data.

\section{Consent for publication}

Not applicable.

\section{Competing interests}

The authors declare that they have no competing interests.

\section{Author details}

${ }^{1}$ Radiology Department, General Hospital "Tzanio", Zanni \& Afentouli 1 Str., 18536 Piraeus, Greece. 'Department of Surgery, General Hospital "Tzanio", Zanni \& Afentouli 1 Str., 18536 Piraeus, Greece. ${ }^{3}$ Urology Department, General Hospital "Tzanio", Zanni \& Afentouli 1 Str., 18536 Piraeus, Greece. ${ }^{4}$ 2nd Department of Radiology, University of Athens, "Attikon" Hospital, Rimini 1 Str., Chaidari, 12462 Athens, Greece.

Received: 25 November 2020 Accepted: 27 January 2021

Published online: 09 February 2021

\section{References}

Andrade G, Khoury HJ, Garzón WJ et al (2017) Radiation exposure of patients and interventional radiologists during prostatic artery embolization: a prospective single-operator study. J Vasc Interv Radiol 28:517-521

Bilhim T, Pisco JM, Rio Tinto H et al (2012a) Prostatic arterial supply: anatomic and imaging findings relevant for selective arterial embolization. J Vasc Interv Radiol 23:1403-1415

Bilhim T, Tinto HR, Fernandes L, Martins Pisco J (2012b) Radiological anatomy of prostatic arteries. Tech Vasc Interv Radiol 15:276-285

Bilhim T, Pisco J, Rio Tinto H et al (2013) Unilateral versus bilateral prostatic arterial embolization for lower urinary tract symptoms in patients with prostate enlargement. Cardiovasc Intervent Radiol 36:403-411

Carnevale FC, Moreira AM, Antunes AA (2014) The "PErFecTED technique": proximal embolization first, then embolize distal for benign prostatic hyperplasia. Cardiovasc Intervent Radiol 37:1602-1605

Carnevale FC, Soares GR, de Assis AM, Moreira AM, Harward SH, Cerri GG (2017) Anatomical variants in prostate artery embolization: a pictorial essay. Cardiovasc Intervent Radiol 40:1321-1337

de Assis AM, Moreira AM, de Paula Rodrigues VC et al (2015) Pelvic arterial anatomy relevant to prostatic artery embolisation and proposal for angiographic classification. Cardiovasc Intervent Radiol 38:855-861

Do KH (2016) General principles of radiation protection in fields of diagnostic medical exposure. J Korean Med Sci 31 Suppl 1(Suppl 1):6-9

Eldem FG, Atak F, Öcal O, Bozaci AC, GÜdeloĞlu A, PeynircloĞlu B (2020) Angiographic prostatic arterial anatomy in Turkish population with benign prostatic hyperplasia. Turk J Med Sci. https://doi.org/10.3906/sag-2004-289

Enderlein GF, Lehmann T, von Rundstedt FC et al (2020) Prostatic artery embolization-anatomic predictors of technical outcomes. J Vasc Interv Radio 31:378-387

Garzón WJ, Andrade G, Dubourcq F et al (2016) Prostatic artery embolization: radiation exposure to patients and staff. J Radiol Prot 36:246-254

Hacking N, Vigneswaran G, Maclean D et al (2019) Technical and imaging outcomes from the UK registry of prostate artery embolization (UK-ROPE) study: focusing on predictors of clinical success. Cardiovasc Intervent Radiol 42:666-676

Hertault A, Maurel B, Midulla M et al (2015) Editor's choice - minimizing radiation exposure during endovascular procedures: basic knowledge, literature review, and reporting standards. Eur J Vasc Endovasc Surg 50:21-36
Hertault A, Rhee R, Antoniou GA et al (2018) Radiation dose reduction during EVAR: results from a prospective multicentre study (the REVAR study). Eur J Vasc Endovasc Surg 56:426-433

Ingwersen M, Drabik A, Kulka U et al (2013) Physicians' radiation exposure in the catheterization lab: does the type of procedure matter? JACC Cardiovasc Interv 6:1095-1102

Lintin L, Barge T, Boardman P, Tong G, Tapping C (2020) Predictors of technical outcome for prostatic artery embolisation using pre-procedural $\mathrm{CT}$ angiography. Eur Radiol. https://doi.org/10.1007/s00330-020-07244-3

Moschouris H, Dimakis A, Anagnostopoulou A, Stamatiou K, Malagari K (2020) Sonographic evaluation of prostatic artery embolization: far beyond size measurements. World J Radiol 12:172-183

Moschouris H, Stamatiou K, Malagari K et al (2019) The value of contrastenhanced ultrasonography in detection of prostatic infarction after prostatic artery embolization for the treatment of symptomatic benign prostatic hyperplasia. Diagn Interv Radiol 25:134-143

Pisco JM, Bilhim T, Pinheiro LC et al (2016) Medium- and long-term outcome of prostate artery embolization for patients with benign prostatic hyperplasia: results in 630 patients. J Vasc Interv Radiol 27:1115-1122

Sukupova L, Hlavacek O, Vedlich D (2018) Impact of the ceiling-mounted radiation shielding position on the Physician's dose from scatter radiation during interventional procedures. Radiol Res Pract. https://doi.org/10.1155/ 2018/4287973

Zumstein V , Binder J, Güsewell S et al (2020) Radiation exposure during prostatic artery Embolisation: A Systematic Review and Calculation of Associated Risks. Eur Urol Focus. https://doi.org/10.1016/j.euf.2020.04.012

\section{Publisher's Note}

Springer Nature remains neutral with regard to jurisdictional claims in published maps and institutional affiliations.

\section{Submit your manuscript to a SpringerOpen ${ }^{\circ}$ journal and benefit from}

- Convenient online submission

- Rigorous peer review

- Open access: articles freely available online

- High visibility within the field

- Retaining the copyright to your article

Submit your next manuscript at $>$ springeropen.com 